\title{
Suppressing anisoplanatism effects in speckle interferometry
}

\author{
C. Stelzer ${ }^{1}$ and H. Ruder ${ }^{2}$ \\ ${ }^{1}$ Institut für Theoretische Astrophysik, Universität Tübingen, Auf der Morgenstelle 10, 72076 Tübingen, Germany \\ e-mail: stelzer@tat.physik.uni-tuebingen.de \\ 2 Institut für Theoretische Astrophysik, Universität Tübingen, Hintere Grabenstr. 30, 72070 Tübingen, Germany
}

Received 31 May 2007 / Accepted 27 August 2007

\section{ABSTRACT}

\begin{abstract}
Aims. The effect of anisoplanatism in speckle interferometry is studied with emphasis on the autocorrelation function and KnoxThompson data.

Methods. We introduce a method for eliminating anisoplanatism artefacts by statistical analysis of Fourier amplitudes calculated from Knox-Thompson data.

Results. With this method, it is possible to suppress anisoplanatism artefacts nearly completely.
\end{abstract}

Key words. techniques: image processing - atmospheric effects - techniques: interferometric - methods: data analysis

\section{Introduction}

Speckle interferometry is widely used for eluding the blurring effect of atmospheric turbulence in stellar imaging. In the beginning, Labeyrie (1970) obtained the unblurred diffraction-limited autocorrelation function from a series of short exposured images. With $\boldsymbol{u}$ the spacial frequency vector (apart from the speckle transfer function, which is a smooth function of $|\boldsymbol{u}|$; Fried 1966; Korff 1973) the Fourier transform of the autocorrelation function is

$\left\langle\left|I_{n}(\boldsymbol{u})\right|^{2}\right\rangle=\left\langle I_{n}(\boldsymbol{u}) I_{n}^{*}(\boldsymbol{u})\right\rangle_{n}$

where $I_{n}(\boldsymbol{u})$ is the Fourier transform of a single speckle interferogram and the mean value over $n$ is taken, and $\langle\ldots\rangle$ denotes the ensemble average. True images were obtained by several methods, e.g. speckle masking (Lohmann et al. 1983), triplecorrelation and bispectra (Bartelt et al. 1984), phase gradient (Aitken et al. 1986), and the method of Knox and Thompson (1974).

This paper is mainly concerned with the Knox-Thompson method where the phase of the spacial frequency components is reconstructed from

$\left\langle I_{n}^{\mathrm{KT}}(\boldsymbol{u}, \Delta \boldsymbol{u})\right\rangle:=\left\langle I_{n}(\boldsymbol{u}) I_{n}^{*}(\boldsymbol{u}+\Delta \boldsymbol{u})\right\rangle_{n}$

with $\Delta \boldsymbol{u}$ a frequency displacement vector. Similar to this is the triple correlation method, where the phase is reconstructed from the bispectrum given by

$\left\langle I_{n}^{\mathrm{TC}}(\boldsymbol{u}, \Delta \boldsymbol{u})\right\rangle:=\left\langle I_{n}(\boldsymbol{u}) I_{n}^{*}(\boldsymbol{u}+\Delta \boldsymbol{u}) I_{n}(\Delta \boldsymbol{u})\right\rangle_{n}$.

In both methods, the phase $\varphi$ is reconstructed recursively with data for several $\Delta \boldsymbol{u}$ using the identities $\varphi^{\mathrm{KT}}(\boldsymbol{u}, \Delta \boldsymbol{u})=\varphi(\boldsymbol{u})-$ $\varphi(\boldsymbol{u}+\Delta \boldsymbol{u})$ and $\varphi^{\mathrm{TC}}(\boldsymbol{u}, \Delta \boldsymbol{u})=\varphi(\boldsymbol{u})-\varphi(\boldsymbol{u}+\Delta \boldsymbol{u})+\varphi(\Delta \boldsymbol{u})$. The triple correlation method has the advantage of taking a real phase closure (Roddier 1986; Ayers et al. 1988), which makes it usable for $|\Delta \boldsymbol{u}|>r_{0}$ ( $r_{0}$ : correlation length of the turbulent atmosphere), whereas the Knox-Thomson method only works for $|\Delta \boldsymbol{u}|<=r_{0}$. However, a combination of these two methods is possible.
A severe problem in speckle interferometry is that it is only feasible within the isoplanatic patch (Roddier et al. 1982) where the intensity of the $n$th image $i_{n}(\boldsymbol{x})$ is approximately given by a convolution of the true image $o(\boldsymbol{x})$ with the point-spread function $p_{n}(\boldsymbol{x})$. In general the point-spread function, the speckle pattern for a point-source, depends on the source coordinates $\boldsymbol{x}^{\prime}$, and the intensity is given by

$i_{n}(\boldsymbol{x})=\int o\left(\boldsymbol{x}^{\prime}\right) p_{n}\left(\boldsymbol{x}-\boldsymbol{x}^{\prime}, \boldsymbol{x}^{\prime}\right) \mathrm{d}^{2} \boldsymbol{x}^{\prime}$

If the image size is larger than a few arc-seconds the space variance of the speckle pattern can not be neglected.

\section{The influence of anisoplanatism on Fourier amplitudes and Knox-Thompson data}

When modelling anisoplanatism we assume that each image $i_{n}(x)$ is a convolution of the object $o(x)$ with a mixture of two point-source speckle interferograms $p_{n, 1}(x)$ and $p_{n, 2}(x)$, weighted with $g_{1}(x)$ and $g_{2}(x)$

$i_{n}(x)=\left[o(x) g_{1}(x)\right] \otimes p_{n, 1}(x)+\left[o(x) g_{2}(x)\right] \otimes p_{n, 2}(x)$.

For the weights, we use $g_{1}(x)=x$ and $g_{2}(x)=1-x$ for $x=0 \ldots 1$ with the Fourier transforms

$G_{1}(u)=\left\{\begin{array}{ll}1 / 2 & \text { for } u=0 \\ \frac{-i}{2 \pi u} & \text { otherwise }\end{array} \quad G_{2}(u)= \begin{cases}1 / 2 & \text { for } u=0 \\ \frac{+i}{2 \pi u} & \text { otherwise. }\end{cases}\right.$

Here we take the space frequency $u$ as a discrete variable. For the sake of simplicity, the calculations are done for one dimension. The treatment of the two-dimensional case is straightforward. This model is a good approximation for small displacements and space frequencies that are not too large. (The fact that the higher frequencies are more affected by anisoplanatism due to the smaller overlap of the responsible aperture areas is not taken in account; see Fig. 3 of Korff (1973) and imagine that the phase 

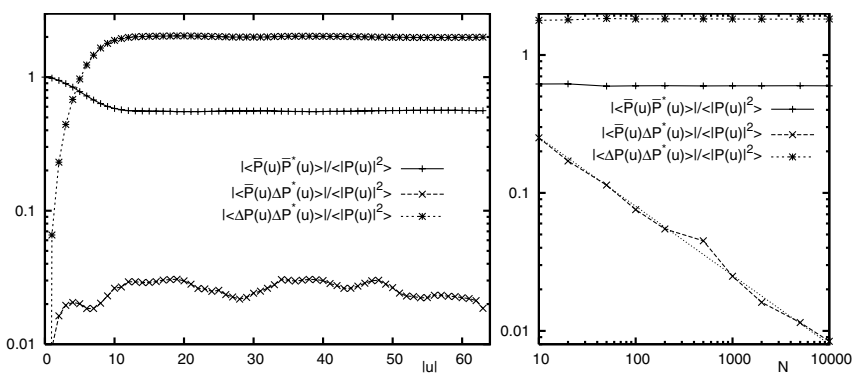

Fig. 1. Examination of the ensemble averages of the pre-factors $\bar{P}_{n}(u) \bar{P}_{n}^{*}(u+\Delta u), \bar{P}_{n}(u) \Delta P_{n}^{*}(u+\Delta u)$, and $\Delta P_{n}(u) \Delta P_{n}^{*}(u+\Delta u)$ in Eq. (8) for $\Delta u=0$ with simulations (2-dimensional). Absolute values are divided by the speckle transfer function $\left\langle|P(\boldsymbol{u})|^{2}\right\rangle$. Left: mean values of $N=1000$ speckle interferograms, plotted against $|\boldsymbol{u}|$ (radial binned). Right: mean values of all $|\boldsymbol{u}|$ and different $N$, plotted against $N$. The dotted line is proportional to $1 / \sqrt{N}$. (Seeing: $r_{0} / D=0.2$, size: $128 \times 128$.)

pattern in the aperture circles is shifted by a fixed amount.) The Fourier transform of the image $i_{n}(x)$ is thus (see Appendix A)

$$
\begin{aligned}
I_{n}(u)= & \frac{1}{2}\left[P_{n, 1}(u)+P_{n, 2}(u)\right] O(u) \\
& +\frac{i}{2 \pi}\left[P_{n, 2}(u)-P_{n, 1}(u)\right] \sum_{u^{\prime} \neq 0} \frac{1}{u^{\prime}} O\left(u-u^{\prime}\right) .
\end{aligned}
$$

With $\bar{P}_{n}(u)=\left[P_{n, 1}(u)+P_{n, 2}(u)\right] / 2$ and $\Delta P_{n}(u)=P_{n, 2}(u)-$ $P_{n, 1}(u)$, the Knox-Thompson data for the frequency shift $\Delta u$ are

$$
\begin{aligned}
& I_{n}^{\mathrm{KT}}(u, \Delta u)=\bar{P}_{n}(u) \bar{P}_{n}^{*}(u+\Delta u) O(u) O^{*}(u+\Delta u) \\
& \quad-\frac{i}{2 \pi} \bar{P}_{n}(u) \Delta P_{n}^{*}(u+\Delta u) \sum_{u^{\prime \prime} \neq 0} \frac{O(u) O^{*}\left(u+\Delta u-u^{\prime \prime}\right)}{u^{\prime \prime}} \\
& \quad+\frac{i}{2 \pi} \Delta P_{n}(u) \bar{P}_{n}^{*}(u+\Delta u) \sum_{u^{\prime} \neq 0} \frac{O\left(u-u^{\prime}\right) O^{*}(u+\Delta u)}{u^{\prime}} \\
& \quad+\frac{1}{4 \pi^{2}} \Delta P_{n}(u) \Delta P_{n}^{*}(u+\Delta u) \sum_{u^{\prime} \neq 0} \sum_{u^{\prime \prime} \neq 0} \frac{O\left(u-u^{\prime}\right) O^{*}\left(u+\Delta u-u^{\prime \prime}\right)}{u^{\prime} u^{\prime \prime}} .
\end{aligned}
$$

The squared amplitudes of the intensity are $\left|I_{n}(u)\right|^{2}=$ $I_{n}^{\mathrm{KT}}(u, \Delta u=0)$. As realised by simulation the mean of $\bar{P}_{n}(u) \Delta P_{n}^{*}(u+\Delta u)$ and $\Delta P_{n}(u) \bar{P}_{n}^{*}(u+\Delta u)$, taken over $N$ images, vanishes for every $u$ and $\Delta u$ proportional to $1 / \sqrt{N}$, see the righthand side of Fig. 1 for an example. For the higher $u$, this is because the Fourier components of two point-source speckle interferograms are mutually uncorrelated. This is the case if the phase structure function (see e.g. Fried 1966) exceeds $2 \pi$ for approximately $u>r_{0}$. The sum and the difference are then obviously uncorrelated, too. For lower $u$, the difference $\Delta P_{n}(u)$ is small, since then the $P_{n, i}(u)(i=1,2)$ have similar values. On the other hand, the mean $\left\langle\Delta P_{n}(u) \Delta P_{n}^{*}(u+\Delta u)\right\rangle_{n}$ for large $N$ is only small compared to $\left\langle\bar{P}_{n}(u) \bar{P}_{n}^{*}(u+\Delta u)\right\rangle_{n}$ if $u$ or $u+\Delta u$ is small. For $u$ and $u+\Delta u$ greater than the long exposure cut-off frequency $r_{0}$, both mean values are of the same order (see Figs. 1 and 2). An explanation is that $\Delta P_{n}(u)$ has the same statistical properties in the uncorrelated regime as the amplitudes of a point-source speckle interferogram multiplied by $\sqrt{2}$. (The $\bar{P}_{n}(u)$ are damped by a factor $1 / \sqrt{2}$.) For large $N$, the ensemble averages of the KnoxThompson data are thus

$$
\begin{aligned}
& \left\langle I_{n}^{\mathrm{KT}}(u, \Delta u)\right\rangle_{n}=\left\langle\bar{P}_{n}(u) \bar{P}_{n}^{*}(u+\Delta u)\right\rangle_{n} O(u) O^{*}(u+\Delta u) \\
& +\frac{\left\langle\Delta P_{n}(u) \Delta P_{n}^{*}(u+\Delta u)\right\rangle_{n}}{4 \pi^{2}} \sum_{u^{\prime} \neq 0} \sum_{u^{\prime \prime} \neq 0} \frac{O\left(u-u^{\prime}\right) O^{*}\left(u+\Delta u-u^{\prime \prime}\right)}{u^{\prime} u^{\prime \prime}},
\end{aligned}
$$

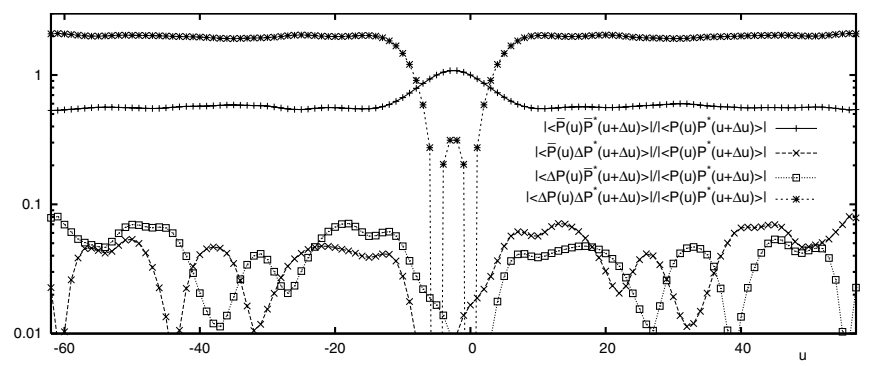

Fig. 2. Examination of the ensemble averages of the pre-factors in Eq. (8) for $\Delta \boldsymbol{u}=(5,0)$ with simulations (2-dimensional). The Slice $\boldsymbol{u}=(u, 0)$ is plotted against $u . N=1000$. Details: see Fig. 1 .

and the mean squared amplitudes are

$$
\begin{aligned}
\left\langle\left|I_{n}(u)\right|^{2}\right\rangle_{n}= & \left\langle\left|\bar{P}_{n}(u)\right|^{2}\right\rangle_{n}|O(u)|^{2} \\
& +\frac{\left\langle\left|\Delta P_{n}(u)\right|^{2}\right\rangle_{n}}{4 \pi^{2}} \sum_{u^{\prime} \neq 0} \sum_{u^{\prime \prime} \neq 0} \frac{O\left(u-u^{\prime}\right) O^{*}\left(u-u^{\prime \prime}\right)}{u^{\prime} u^{\prime \prime}} .
\end{aligned}
$$

Here one can see that some spurious inter-modulation products appear except for the Fourier components of the object. They cause the granular look of a traditional reconstruction, often with spurious structures and ghost images of the object. Sometimes the granularity hides the original object almost completely. See the lefthand side of Figs. 3 and 5.

\section{Knox-Thompson triples for calculating the Fourier amplitudes}

Now we present a new method with which we try to overcome the effects of anisoplanatism in conventional speckle interferometry. It is also possible to calculate the mean squared Fourier amplitudes from a triple of mean Knox-Thompson data. With $I^{\mathrm{KT}}(\boldsymbol{u}, \Delta \boldsymbol{u})=\left\langle I_{n}^{\mathrm{KT}}(\boldsymbol{u}, \Delta \boldsymbol{u})\right\rangle_{n}$ for the frequency shifts $\Delta \boldsymbol{u}_{1}, \Delta \boldsymbol{u}_{2}$ and $\Delta \boldsymbol{u}_{3}=\Delta \boldsymbol{u}_{2}-\Delta \boldsymbol{u}_{1}$, the mean squared amplitudes $|I(\boldsymbol{u})|^{2}=$ $\left\langle\left|I_{n}(\boldsymbol{u})\right|^{2}\right\rangle_{n}$ are given by

$|I(\boldsymbol{u})|^{2}=\mathfrak{R}\left\{\frac{I^{\mathrm{KT}}\left(\boldsymbol{u}, \Delta \boldsymbol{u}_{1}\right) I^{K T *}\left(\boldsymbol{u}, \Delta \boldsymbol{u}_{2}\right)}{I^{K T *}\left(\boldsymbol{u}+\Delta \boldsymbol{u}_{1}, \Delta \boldsymbol{u}_{3}\right)}\right\}$.

Having Knox-Thompson data for several hundred frequency shifts $\Delta \boldsymbol{u}$, one finds several thousand triples to calculate each amplitude. Inter-modulations come in from different object amplitudes for different frequency shifts, as seen from the last term of Eq. (9). In the mean value of the amplitude, they are mostly averaged out. But in general, due to noise, the phases of the Knox-Thompson data for the higher frequencies $\boldsymbol{u}$ are strongly varying. (For a good phase reconstruction, one needs the average phase of a lot of Knox-Thompson data with different frequency shifts.) This causes a damping of the higher Fourier amplitudes, resulting in a blurred reconstruction. To avoid this damping, we calculate the mean squared amplitudes from

$|I(\boldsymbol{u})|^{2}=\frac{\left|I^{\mathrm{KT}}\left(\boldsymbol{u}, \Delta \boldsymbol{u}_{1}\right)\right|\left|I^{\mathrm{KT}}\left(\boldsymbol{u}, \Delta \boldsymbol{u}_{2}\right)\right|}{\left|I^{\mathrm{KT}}\left(\boldsymbol{u}+\Delta \boldsymbol{u}_{1}, \Delta \boldsymbol{u}_{3}\right)\right|}$.

Let us denote the mean value of these results of all KnoxThompson triples by $\left|I_{\mathrm{tr}}(\boldsymbol{u})\right|^{2}=\left\langle|I(\boldsymbol{u})|^{2}\right\rangle_{\mathrm{tr}}$ and the variance by $\sigma_{\text {tr }}^{2}(\boldsymbol{u})=\left\langle|I(\boldsymbol{u})|^{4}\right\rangle_{\mathrm{tr}}-\left\langle|I(\boldsymbol{u})|^{2}\right\rangle_{\mathrm{tr}}^{2}$. A reconstruction on the basis of the $\left|I_{\mathrm{tr}}(\boldsymbol{u})\right|^{2}$ shows less anisoplanatism artefacts than the traditional one, as can be seen in the middle part of Fig. 3. But some 

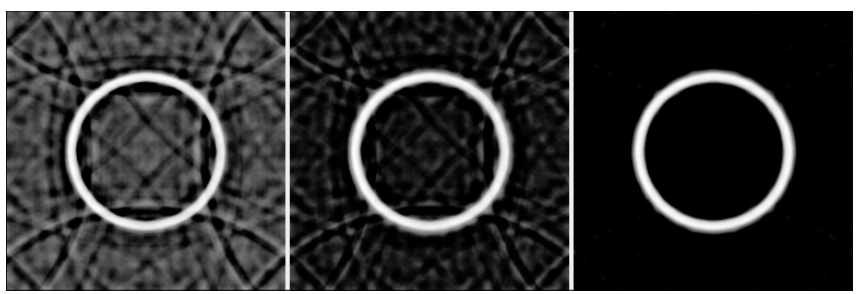

Fig. 3. Reconstruction of a ring-shaped object from 1000 simulated anisoplanatic speckle interferograms with 100 Knox-Thompson data sets. Left: traditional reconstruction; middle: reconstruction with amplitudes from Eq. (12); right: with amplitudes from Eq. (18). (Seeing: $r_{0} / D=0.1$, isoplanatic length: 10 pixel, one turbulence layer, size: $256 \times 256$ pixel.)

artefacts are still present with an intensity of, rough estimated, a quarter of the former. Artefacts very near to the object were nearly undamped. Also in the reconstruction process for Fig. 6, calculating the amplitudes in this way does not yet lead to a satisfactory result. In the following, a better method for compensation for the anisoplanatism effect is presented.

Now let us assume that the inter-modulation products influencing the term in the bracket of Eq. (11) are Gaussian distributed in the complex plane $x, i y$. With $\mu$ the true value of the squared amplitude and $\sigma^{2}$ the variance, we have the probability density function:

$P(x, y)=\frac{1}{2 \pi \sigma^{2}} \exp \left[-\frac{(x-\mu)^{2}+y^{2}}{2 \sigma^{2}}\right]$.

If taking absolute values $r=\left(x^{2}+y^{2}\right)^{1 / 2}$ as done in Eq. (12), the probability density function is

$P(r)=\frac{r}{\sigma^{2}} \exp \left[-\frac{r^{2}+\mu^{2}}{2 \sigma^{2}}\right] J_{0}\left(\frac{i \mu r}{\sigma^{2}}\right)$

where $J_{0}$ is the Bessel function of the first kind of zero order (Abramowitz \& Stegun 1970). The mean value of $r$ is thus

$\langle r\rangle=\sigma \sqrt{2} \exp \left[-\frac{\mu^{2}}{2 \sigma^{2}}\right] \sum_{l=0}^{\infty}\left(\frac{\mu^{2}}{2 \sigma^{2}}\right)^{l} \frac{\Gamma(l+3 / 2)}{(l !)^{2}}$.

For our purpose Eq. (15) is well approximated by

$\langle r\rangle \approx \mu\left[1+\frac{\pi \sigma^{2}}{2 \mu^{2}}\right]^{1 / 2}$,

which was realised by inspecting the plots: in the interesting area of the parameters $\mu$ and $\sigma$ the difference is less than 5 percent. For $\mu=0$ (and probably for $\mu / \sigma \rightarrow \infty$ ), Eq. (16) is exact. The second-order moment is

$\left\langle r^{2}\right\rangle=\int_{0}^{\infty} r^{2} P(r) \mathrm{d} r=\mu^{2}+2 \sigma^{2}$.

With the approximation of $\langle r\rangle$ and $\sigma_{r}^{2}=\left\langle r^{2}\right\rangle-\langle r\rangle^{2}$, we obtain (see Appendix B)

$\mu=\langle r\rangle\left[1-\frac{\pi}{4-\pi} \cdot \frac{\sigma_{r}^{2}}{\langle r\rangle^{2}}\right]^{1 / 2}$.

Identifying $\langle r\rangle$ with $\left|I_{\mathrm{tr}}(\boldsymbol{u})\right|^{2}$ and $\sigma_{r}^{2}$ with $\sigma_{\mathrm{tr}}^{2}(\boldsymbol{u})$, we get a better approximation with $\mu$ for the true $|I(\boldsymbol{u})|^{2}$. (In practice, we multiply the $\sigma_{\text {tr }}^{2}(\boldsymbol{u})$ with a correction factor near unity to compensate for the influence of the different damping factors

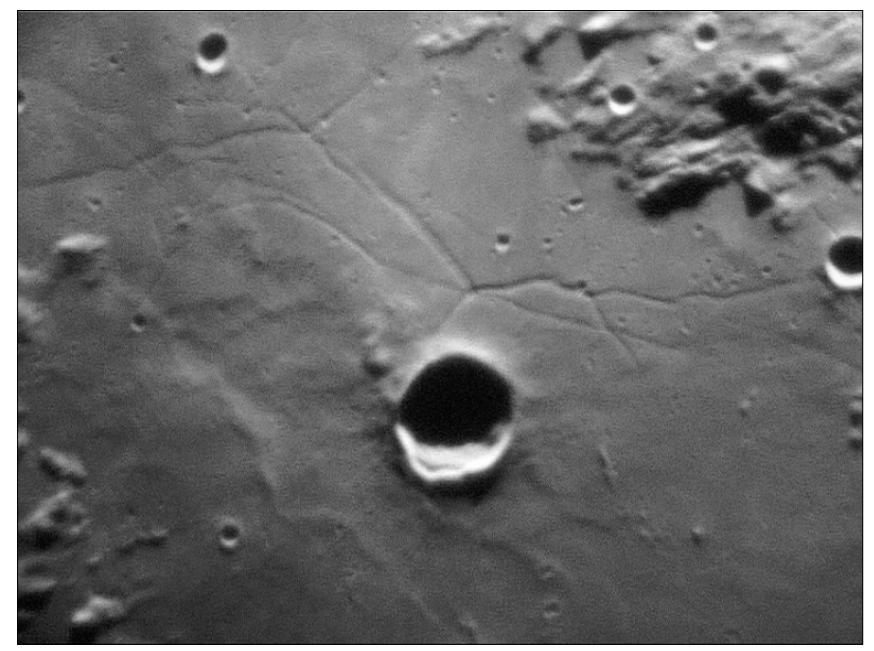

Fig. 4. Example of one raw speckle interferogram from a part of the moon with which we reconstruct Figs. 5 and 6. Exposure time was $3 \mathrm{~ms}$. Field of view was $90 \times 70$ arcsec. Pixel size was 0.12 arcsec. (Original size is $720 \times 576$ pixel and is scaled for the correct aspect ratio to $768 \times$ 576 pixel.)

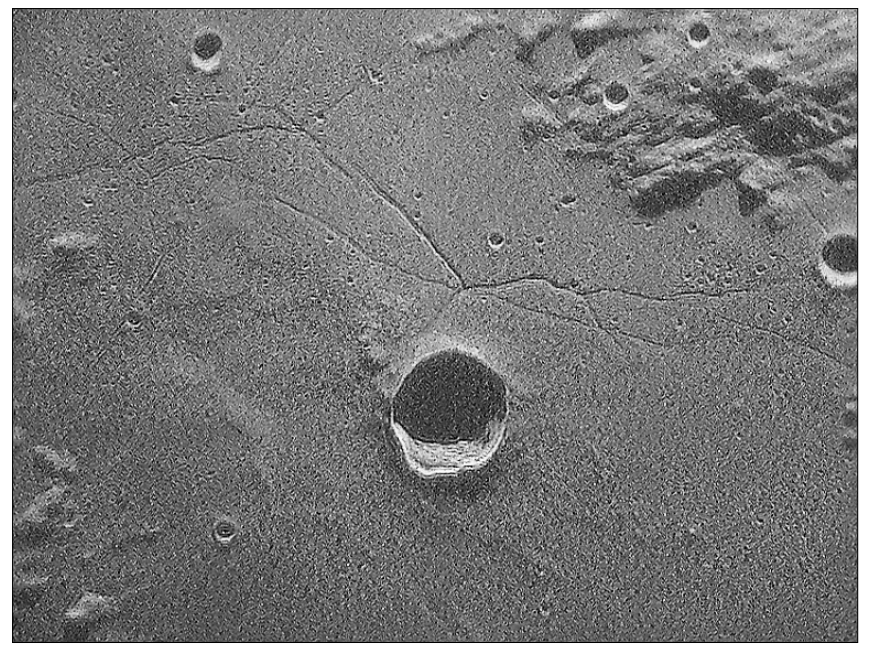

Fig. 5. Traditional Knox-Thompson reconstruction from a series of 923 speckle interferograms like the one shown in Fig. 4. (Field of view and size equals those of Fig. 4, reconstruction details: see Sect. 4.)

$\left\langle P_{n}(\boldsymbol{u}) P_{n}^{*}(\boldsymbol{u}+\Delta \boldsymbol{u})\right\rangle_{n}$ for the Knox-Thompson data with different frequency shifts $\Delta \boldsymbol{u}$.) With this amplitude, one gets a reconstruction where almost all anisoplanatism artefacts are removed without the blurring of the former method (see the righthand side of Figs. 3 and 6). With some modifications (i.e. prefixing the $\left.|I(\Delta \boldsymbol{u})|^{2}\right)$, the method also works with triple correlation data if only data with small $|\Delta \boldsymbol{u}|$ or small $|\boldsymbol{u}+\Delta \boldsymbol{u}|$ are considered.

\section{Application of the method}

As an example in Fig. 6, a reconstruction with the method described in the previous section of the image of the moon crater Triesenecker is shown. This reconstruction is based on a series of about a thousand speckle interferograms recorded at the $60 \mathrm{~cm}$ telescope of the Capella-Observatory in Namibia with a Sony camcorder. One example is given in Fig. 4. The size of the Airy disc 0.23 arcsec equals 1.9 pixel. The Fried parameter for the seeing is estimated to $r_{0}=10 \mathrm{~cm}$ with a seeing disc 1.25 arcsec. The speckle interferograms were first aligned and 


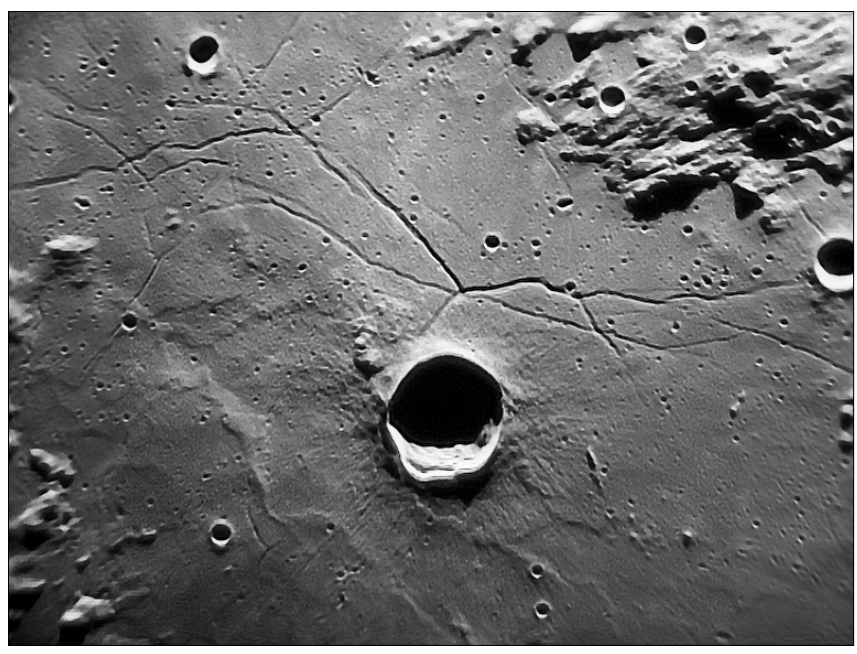

Fig. 6. Reconstruction with our new method, Eq. (18), from the same speckle interferograms with which we reconstruct Fig. 5. (Other reconstruction parameters are identical to those of Fig. 5, details: see Sect. 4.) A part of the moon is shown. The big crater below the centre is Triesenecker.

then inserted in the aligned sum picture, which was larger due to the tip-tilt mode and telescope movements. As a result, we were able to make the reconstruction field of view equal to that of the speckle interferograms (with a slight loss in sharpness near the borders). For the reconstruction, the speckle interferograms were tiled with $4 \times 3$ overlapping sub-images of size $256 \times 256$ pixels. For each sub-image, 400 Knox-Thompson date sets are calculated with frequency shift vectors $\Delta \boldsymbol{u}$ bounded by a semicircle of a radius of about 16. Figure 5 shows the result from a traditional Knox-Thompson reconstruction. Finer structures of the object are visible, but accompanied by anisoplanatism artefacts. Ghost images are present. A high granularity (here partly also due to noise) occurs. Applying our new method, Eq. (18), all Knox-Thompson triples with $\Delta \boldsymbol{u}_{i}<=12(i=1,2,3)$ are used (9368 triples). In the result, Fig. 6, nearly all artefacts are removed. The finest details observable are about pixel size, which means that nearly diffraction-limited resolution is reached.

\section{Conclusion}

We have analysed the effect of anisoplanatism in speckle interferometry by means of a model and found that it is caused from an inter-modulation of the Fourier components. Also, the anisoplanatism effect on the Knox-Thompson data was analysed. We found that the anisoplanatism effects could be reduced by calculating the Fourier amplitudes as mean values from triples of the Knox-Thompson data. The anisoplanatism effects could be mostly suppressed by means of a statistical analysis based on the Knox-Thompson triples. An example of the reconstruction of an extended object is given where the method developed here was successfully applied. (A program can be downloaded from www . tat . physik. uni-tuebingen.de/ stelzer)

\section{Appendix A: Derivation of Eq. (7)}

Taking the Fourier transform of Eq. (5), we get

$$
\begin{aligned}
I_{n}(u)= & {\left[O(u) \otimes G_{1}(u)\right] P_{n, 1}(u) } \\
& +\left[O(u) \otimes G_{2}(u)\right] P_{n, 2}(u)
\end{aligned}
$$

where now $\otimes$ means convolution in $u$. With discrete $u$, we have

$$
\begin{aligned}
I_{n}(u)= & P_{n, 1}(u) \sum_{u^{\prime}} O\left(u-u^{\prime}\right) G_{1}\left(u^{\prime}\right) \\
& +P_{n, 2}(u) \sum_{u^{\prime}} O\left(u-u^{\prime}\right) G_{2}\left(u^{\prime}\right) .
\end{aligned}
$$

Inserting $G_{1}(u)$ and $G_{2}(u)$ from Eq. (6), we get Eq. (7).

\section{Appendix B: Derivation of Eq. (18)}

From the Knox-Thompson triples, we get the mean squared amplitudes $\left|I_{\mathrm{tr}}(\boldsymbol{u})\right|^{2}$, which are identified with $\langle r\rangle$, and their variances $\sigma_{\text {tr }}^{2}(\boldsymbol{u})$, which then equals $\sigma_{r}^{2}$. We are looking for the underlying squared amplitudes $|I(\boldsymbol{u})|^{2}$ of the true object that corresponds to $\mu$ in our statistical model. Now with $\left\langle r^{2}\right\rangle$ from Eq. (17) in $\sigma_{r}^{2}=\left\langle r^{2}\right\rangle-\langle r\rangle^{2}$, we eliminate $\sigma$ in the approximate expression for $\langle r\rangle$, Eq. (16), and obtain Eq. (18) for $\mu$.

\section{References}

Abramowitz, M., \& Stegun, I. 1970, Handbook of Mathematical Functions (National Bureau of Standards)

Aitken, G., Houtman, R., Johnson, R., \& Pochet, J.-M. 1986, Appl. Opt., 24, 2926

Ayers, G., Northcott, M., \& Dainty, J. 1988, J. Opt. Soc. Am. A, 5, 963 Bartelt, H., Lohmann, A., \& Wirnitzer, B. 1984, Appl. Opt., 23, 3121

Fried, D. 1966, J. Opt. Soc. Am., 56, 1372

Knox, K., \& Thompson, B. 1974, AJ, 193, L45

Korff, D. 1973, J. Opt. Soc. Am., 63, 971

Labeyrie, A. 1970, A\&A, 6, 85

Lohmann, A., Weigelt, G., \& Wirnitzer, B. 1983, Appl. Opt., 22, 4028

Roddier, F. 1986, Opt. Comm., 60, 145

Roddier, F., Gilli, J., \& Vernin, J. 1982, J. Opt. (Paris), 13, 63 